\title{
DESIGN AND FABRICATION OF MEDICATED CHOCOLATE FORMULATION BY CHOCOLATE DRUG DELIVERY SYSTEM
}

\author{
REDDY SUNIL ${ }^{* 1}$, K. MOUNIKA ${ }^{2}$, A. VENKATESHAM ${ }^{1}$ \\ 1S. V. S. Group of Institutions, S. V. S. School of Pharmacy, Bheemaram, Warangal, Telangana State. India, 2St. Peter's College of Pharmacy, \\ Madikonda, Kazipet, Hanamkonda. Telangana State India \\ Email: drsunilsvsjntuh@gmail.com \\ Received: 24 May 2017, Revised and Accepted: 22 Jul 2017
}

\begin{abstract}
Objective: The objective of this study is to design and fabricate Chlorpheniramine Maleate chocolate formulation by chocolate drug delivery system. Chlorpheniramine Maleate binds to histamine H1 receptor. This blocks the action of endogenous histamine, which subsequently leads to temporary relief of negative symptoms brought on by histamine.

Methods: Chocolate is a range of products derived from cocoa (cacao), mixed with fat (i.e., cocoa butter) and finely powdered sugar to produce a solid confectionery. The medicated chocolate formulation is widely used for pediatric administration and increases patient compliance. Chlorpheniramine Maleate chocolate formulation is prepared to improve patient compliance. Chocolates were formulated (F1-F3) with a total fat of $25-35 \%(\mathrm{w} / \mathrm{w})$ from cocoa liquor and cocoa butter with more than $34 \%$ total cocoa, composition as specified for dark chocolate, lecithin, sweetening agents.
\end{abstract}

Results: The prepared chocolate formulations were evaluated for general appearance, drug content, In vitro drug release and DSC and FTIR, moisture content and blooming tests. F1 formulation releases complete drug within $60 \mathrm{~min}$.

Conclusion: The results indicate that the formulation has no drug excipient interactions and there was no degradation in drug, it is stable during chocolate formulation preparation.

Keywords: Chlorpheniramine Maleate, Chocolate, Medicated chocolate, In vitro drug release

(c) 2017 The Authors. Published by Innovare Academic Sciences Pvt Ltd. This is an open access article under the CC BY license (http://creativecommons.org/licenses/by/4.0/) DOI: http://dx.doi.org/10.22159/ijcpr.2017v9i5.22156

\section{INTRODUCTION}

Oral route is one of the best routes for patient compliance. It has its own advantages. Per contra, it has its own disadvantages too. Drugs with first pass metabolism cannot be administered through oral route. So, there is a situation to consider absorptive mucosa as the route of administration to administer drugs with first pass metabolism. One of the examples of absorptive mucosa includes Tran's mucosal route as a route of administration. A Tran's mucosal route includes mucosal linings along nasal, vaginal, rectal and oral cavities [1-2].

Chocolate is highly sophisticated a versatile food that is combined to create completely different taste and texture sensations. Chocolate is also an anhydrous medium and is therefore resistant to microbial growth and to hydrolysis of water-sensitive active agents. Chocolate is well-suited as a vehicle for delivering active agents in many aspects. For example, the organoleptic characteristics of chocolate are excellent for masking unpleasant flavors associated with some active agents and giving a smooth and creamy texture to compositions of active agents that are otherwise undesirably gritty. Chocolate is a range of products derived from cocoa (cacao), mixed with fat (i.e., cocoa butter) and finely powdered sugar to produce a solid confectionery.

Medicated chocolate is prepared by using chocolate base and the drug is incorporated into prepared chocolate base [3]. As the drug is incorporated within the chocolate and the drug is released from the chocolate, it is called as Chocolate drug delivery system.

Chocolate drug delivery system has advantages that include a possible bypass of first-pass effects and avoidance of pre-systemic elimination within the GI tract. Chocolate is also an anhydrous medium and is therefore resistant to microbial growth and to hydrolysis of water-sensitive active agents. Chocolate is well-suited as a vehicle for delivering active agents in many aspects.

The aim of present study is to design and formulate a chocolate formulation as a drug delivery system of a first generation alkyl amine Anti-histamine drug Chlorpheniramine Maleate with the following objective:-

- To improve patient compliance.

- To study the kinetic profile of drug release.

- Comparison of release profiles of different composition of excipients in formulations.

In the present study, chocolate drug delivery system through the oral route, especially the buccal route was utilized as a platform for Histamine receptors antagonists; hence it is possible to realize local effect drug administration. In local effect, the aim is to achieve a site specific release of the drug on mucosa, whereas the systemic effect involves drug absorption through the mucosal barrier to reach systemic circulation. The buccal mucosa is highly vascularized and presents a reduced enzymatic activity when compared to gastro intestinal, rectal and nasal mucosa [4].

\section{MATERIALS AND METHODS}

\section{Materials}

Chlorpheniramine Maleate, Lecithin, Pharmaceutical grade sugar was purchased from Nihal traders (Hyderabad, Telangana, India.), AET Labs (Hyderabad, Telangana, India.), respectively Cocoa Butter was purchased from AET Labs (India), Cocoa Powder was purchased from WEIKFIELD India. All the other chemicals like Dicalcium Phosphate, Mango flavor, Pine apple flavor used are of analytical grade.

\section{Methodology}

\section{Formulation of chocolate base}

Chocolates were formulated with a total fat of $25-35 \%(w / w)$ from cocoa liquor and cocoa butter with more than $34 \%$ total cocoa, composition as specified for dark chocolate (table I) [7]. 


\section{Method of preparation}

The oven was set to $50^{\circ} \mathrm{C}$. In a beaker, sugar and water were taken and kept in the oven for 4-5 min and syrup was prepared. Then cocoa butter was taken and kept in the beaker in the oven for $1 \mathrm{~min}$. Then sugar syrup was removed from the oven, and cocoa powder was added and mixed well. Careful attention is paid to the chocolate manufacturing process to ensure that the temperature of the mixture is not too high. Then above mixture of the chocolate base was cooled up to semisolid consistency and then flavor was added [6-9]

\section{Formulation of medicated chocolate}

Chocolate squares containing drug in appropriate quantity is known as medicated chocolate.

\section{Method of preparation}

Oven was set at $50{ }^{\circ} \mathrm{C}$. Then chocolate base was melted till it becomes free flowing liquid. After above step; required quantity of drug was added. Then whole mass was stirred well with the help of magnetic stirrer to ensure uniform mixing. Then we poured the above mixture in a polycarbonate set mould and refrigerated for 15 min till it becomes solid.

Medicated chocolates were prepared in the three formulations. In the I formulation, a batch of ten units was prepared where each single unit possess Chlorpheniramine Maleate drug, Cocoa Powder, Cocoa Butter, Lecithin and Pharmaceutical Grade Sugar were mixed uniformly in a proper order, in order to get a medicated chocolate formulation. But a problem occurred in the sense of odor. While preparing the formulation, the Cocoa powder odor was very much dominating and was strong to smell and while evaluating the formulation, a pungent smell caused by Lecithin was also observed. So, a flavor was added in order to reduce the strong smell and pungent smell of Cocoa powder and Lecithin. The formulation was shown in the table 1.

In the II formulation, a batch of ten units was prepared where each single unit possess Chlorpheniramine Maleate drug, Cocoa Powder, Cocoa Butter, Lecithin, Pharmaceutical Grade Sugar and Pine apple flavor were mixed evenly in a proper order, in order to get a medicated chocolate formulation. Here in this formulation, additionally flavor was added to the formulation in order to mask the strong odor and pungent smell of Cocoa powder and Lecithin.

So, in proper solidification of formulation while collecting the prepared medicated chocolate from the molds and chances of breakage of formulation and there by the improper shaping of the formulation. So, in order to overcome this problem, an adsorbent Dicalcium phosphate was added to the formulation. The formulation was shown in the table 2 .

In the III formulation, a batch of ten formulations were prepared where each single unit possess Chlorpheniramine Maleate drug, Cocoa Powder, Cocoa Butter, Lecithin, Pharmaceutical Grade Sugar, Mango flavor and Dicalcium Phosphate were mixed evenly in a proper order, in order to get a medicated chocolate formulation. The formulation was shown in the table 3 .
Additionally Dicalcium Phosphate was added to the formulation in order to overcome in proper solidification of formulation while collecting the prepared medicated chocolate from the molds. There is a change in the type of flavor used, which is a Mango flavor in formulation III and Pine apple flavor was used in formulation II. Pine apple flavor containing formulation was very much acceptable when compared to Mango flavor. The Pine apple flavor acceptance is very good, in the sense of odor and taste.

\section{Evaluation parameters}

\section{Evaluation of chocolate base}

\section{Taste, texture and mouth feel characteristics assessment}

Taste, texture and mouth feel characteristics of chocolate were evaluated by taking panel of 10 human volunteers on a rating scale of 1-5.

\section{Evaluation of medicated chocolates \\ Evaluation of physicochemical properties \\ Drug excipient compatibility studies}

Drug excipient compatibility tests like DSC and FTIR are performed for both pure drug as well as Medicated Chocolate i.e., Chocolate base with Chlorpheniramine Maleate and other excipients used in the formulation.

\section{Thickness}

The thickness of ten formulations from each batch was determined using Vernier calipers. The thickness variation limits allowed are $\pm 5 \%$ of the size of the formulation.

\section{Weight variation}

Weight Variation study was carried out as per USP. Five formulations were randomly selected from each batch weighed individually. The average weight and standard deviation was calculated.

\section{Disintegration test}

Disintegration Test for the prepared formulation was carried out as per USP until it disintegrates using Disintegration tester (at $37 \pm 0.5^{\circ} \mathrm{C}$ ) and $60 \mathrm{rpm}$ speed using $6.8 \mathrm{pH}$ buffer for $20 \mathrm{~min}$.

\section{Drug content determination}

Drug content of a medicated chocolate was determined by using UV Spectrometer at $260 \mathrm{~nm}$ against blank.

\section{$>$ In vitro drug release studies}

In vitro drug release was studied using USP II apparatus, with $500 \mathrm{ml}$ of dissolution medium maintained at $37 \pm 1{ }^{\circ} \mathrm{C}$ for $1 \mathrm{~h}(5$, $10,15,20,25,30,35,40,45,50,55,60 \mathrm{~min})$, at $50 \mathrm{rpm}$. pH 6.8 Phosphate buffer was used as a dissolution medium. $5 \mathrm{ml}$ of sample was withdrawn in different time intervals and was replaced by an equal volume of fresh dissolution medium of the same $\mathrm{pH}$. Collected samples were analyzed spectrophotometrically at $260 \mathrm{~nm}$, and cumulative percent drug release was calculated. The study was performed in triplicate.

Table 1: Formulation-I

\begin{tabular}{llll}
\hline S. No. & Ingredient & Category \\
\hline 1. & Chlorpheniramine Maleate & Drug & Quantity \\
2. & Cocoa powder & Principle ingredient & $4 \mathrm{mg}$ \\
3. & Cocoa butter & Solidifying agent & $1446 \mathrm{mg}$ \\
4. & Lecithin & Emulsifier & $500 \mathrm{mg}$ \\
\hline
\end{tabular}

Table 2: Formulation-II

\begin{tabular}{llll}
\hline S. No. & Ingredient & Category & \\
\hline 1. & Chlorpheniramine Maleate. & Drug & \\
2. & Cocoa powder & Principle ingredient & \\
3. & Cocoa butter & Solidifying agent & 1350 \\
4. & Lecithin. & Emulsifier & 586 \\
5. & Pharmaceutical grade sugar & Sweetening agent & 45 \\
6. & Pine apple flavour & Flavour & 1000 \\
7. & Total & - & 15 \\
\hline
\end{tabular}


Table 3: Formulation-III

\begin{tabular}{llll}
\hline S. No. & Ingredient & Category & Quantity (mg) \\
\hline 1. & Chlorpheniramine Maleate. & Drug & 4 \\
2. & Cocoa powder & Principle ingredient & 1251 \\
3. & Cocoa butter & Solidifying agent & 500 \\
4. & Lecithin. & Emulsifier & 30 \\
5. & Pharmaceutical grade sugar & Sweetening agent & 1000 \\
6. & Mango flavour & Flavour & 15 \\
7. & Dicalcium phosphate. & Adsorbent & 200 \\
8. & Total & - & 3000 \\
\hline
\end{tabular}

Table 4: Standard graph of chlorpheniramine maleate

\begin{tabular}{lll}
\hline S. No. & Concentration $(\mu \mathrm{g} / \mathbf{m l})$ & Absorbancy $(\mathbf{n m})$ \\
\hline 01. & 5 & 0.129 \\
02. & 10 & 0.186 \\
03. & 15 & 0.239 \\
04. & 20 & 0.296 \\
05. & 25 & 0.357 \\
06. & 30 & 0.405 \\
07. & 35 & 0.455 \\
08. & 40 & 0.505 \\
09. & 45 & 0.549 \\
10. & 50 & 0.586 \\
11. & 60 & 0.689 \\
\hline
\end{tabular}

Table 5: Preparation of chocolate base

\begin{tabular}{llll}
\hline S. No. & Ingredient & Category & Quantity (mg) \\
\hline 1. & Cocoa powder & Principle ingredient. & 1430 \\
2. & Cocoa butter & Solidifying agent. & 500 \\
3. & Lecithin & Emulsifier. & 50 \\
4. & Pharmaceutical grade sugar & Sweetening agent. & 1000 \\
\hline
\end{tabular}

Table 6: Taste, texture and mouth feel characteristics assessment

\begin{tabular}{llll}
\hline S. No. & Character & Criteria & Scale \\
\hline 01. & Appearance & Glossy, even shine, no dots. & \\
02. & Aroma or smell & Chocolaty with only a light scent of any flavorings', fresh with no chemical smell. \\
03. & Snap & Break clean without crumbling or layering. & $1-5$ with 5 being the best. \\
04. & Taste & Chocolaty, flavors not over powder the chocolate taste. Good after taste. & $1-5$ with 5 being the best. \\
05. & Texture & Creamy being the best. & $1-5$ with 5 being the best. \\
\hline
\end{tabular}

Table 7: General appearance

\begin{tabular}{lll}
\hline S. No. & Characteristics & Result \\
\hline 01. & Color & Dark brown \\
02. & Odor & Pleasant \\
03. & Taste & Semisweet \\
04. & Texture & Smooth \\
\hline
\end{tabular}

Table 8: Thickness of medicated chocolate formulations

\begin{tabular}{ll}
\hline Formulation & Thickness (mm) \\
\hline F1 & $8.99 \pm 0.02$ \\
F2 & $8.36 \pm 0.09$ \\
F3 & $9.18 \pm 0.05$ \\
\hline
\end{tabular}

Table 9: Weight variation of medicated chocolate formulations

\begin{tabular}{ll}
\hline Formulation & Weight variation (gms) \\
\hline F1 & $3.06 \pm 0.24$ \\
F2 & $3.02 \pm 0.30$ \\
F3 & $3.10 \pm 0.28$ \\
\hline
\end{tabular}

Table 10: Disintegration of medicated chocolate formulations

\begin{tabular}{ll}
\hline Formulation & Disintegration (min) \\
\hline F1 & $20 \pm 2$ \\
F2 & $22 \pm 1$ \\
F3 & $23 \pm 4$ \\
\hline
\end{tabular}


Sunil et al.

Table 11: Drug content of medicated chocolate formulations

\begin{tabular}{ll}
\hline Formulation & Drug content (\%) \\
\hline F1 & $94.83 \pm 0.71$ \\
F2 & $98.49 \pm 0.54$ \\
F3 & $95.51 \pm 0.33$ \\
\hline
\end{tabular}

Table 12: In vitro drug release studies of medicated chocolate formulations

\begin{tabular}{llll}
\hline Time (min) & F1 & F2 & \\
\hline 0 & 0 & 0 & \\
5 & 25.10 & 30.05 & 3 \\
10 & 39.62 & 42.62 & 45.15 \\
15 & 51.66 & 66.88 & 67.85 \\
20 & 75.06 & 79.09 & 84.66 \\
30 & 89.05 & 98.05 & 99.05 \\
45 & 99.00 & 100.99 & 101.62 \\
60 & 100.00 & -- & -- \\
\hline
\end{tabular}

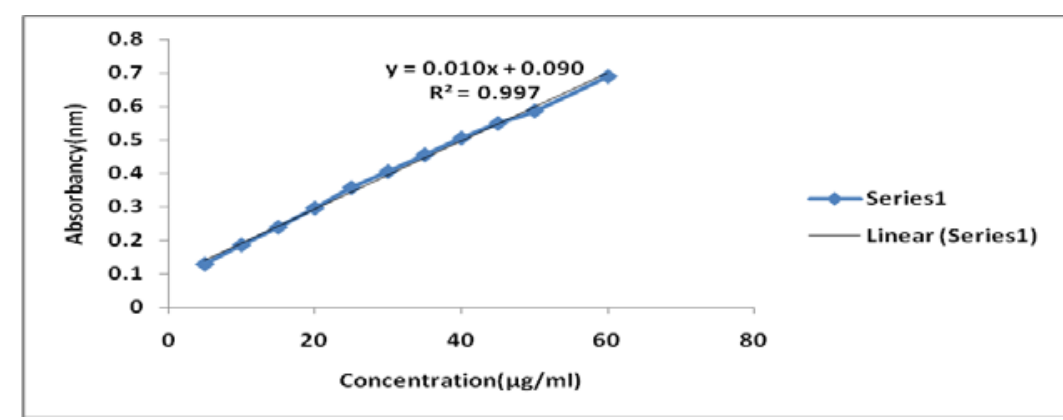

Fig. 1: Standard graph of chlorpheniramine maleate in $6.8 \mathrm{pH}$ buffer

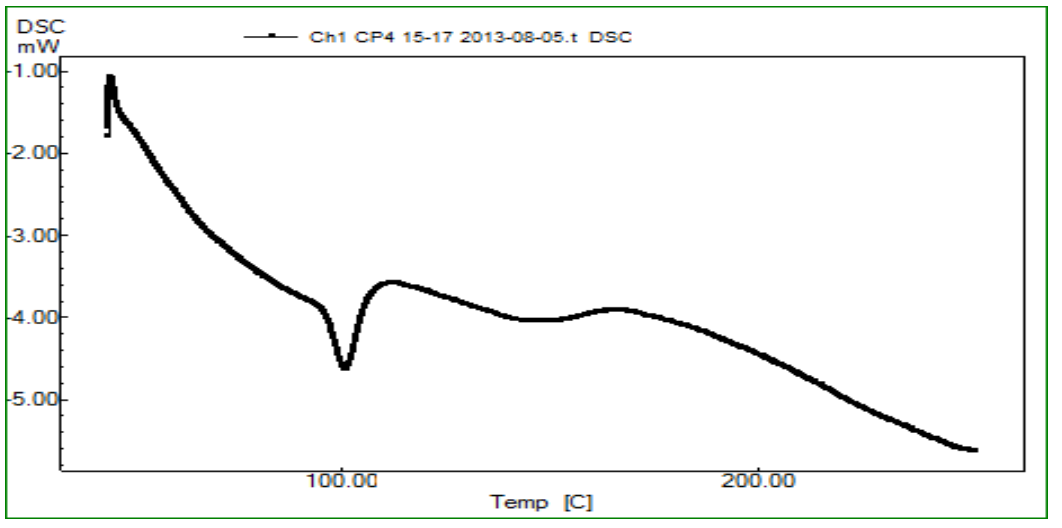

Fig. 2: DSC spectra of chlorpheniramine maleate

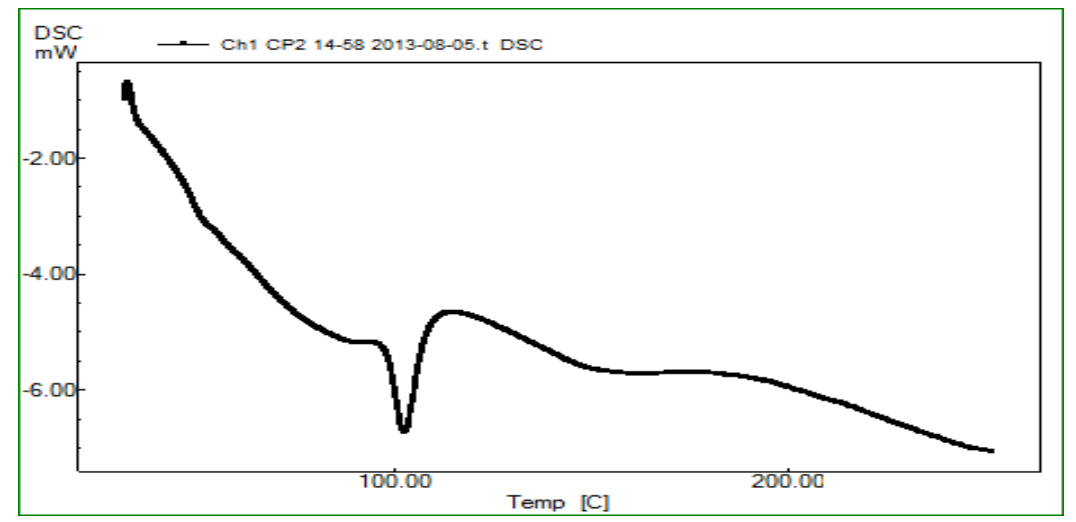

Fig. 3: DSC spectra of medicated chocolate (chlorpheniramine maleate+chocolate base) 


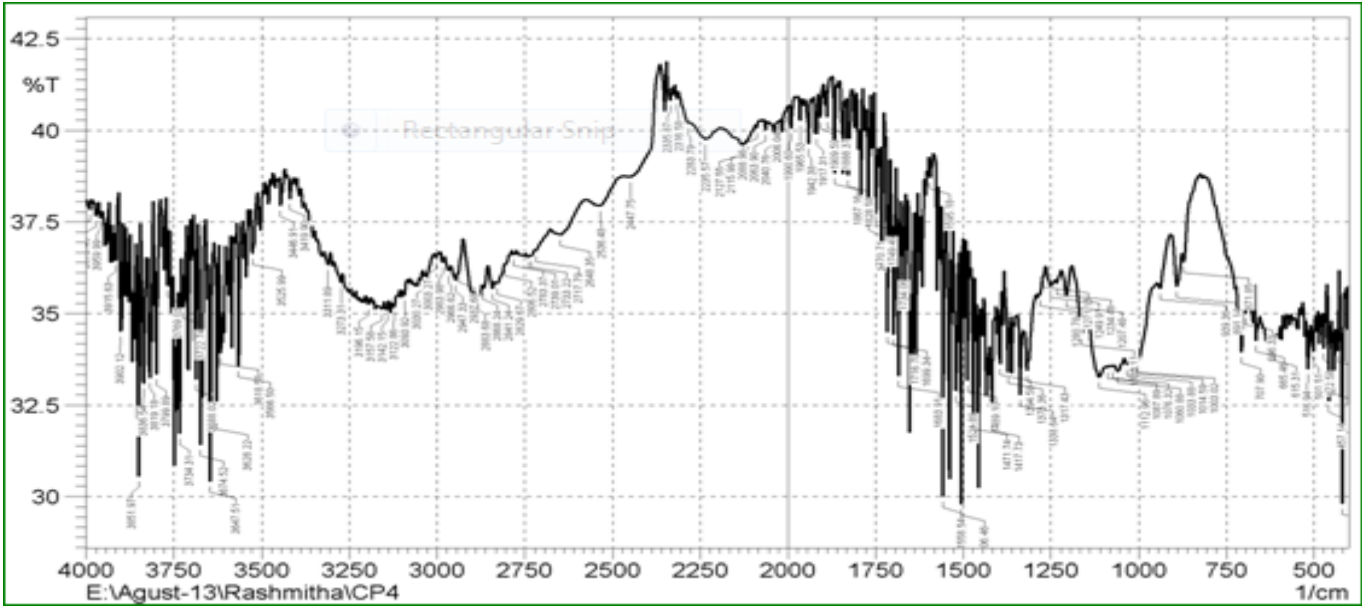

Fig. 4: FTIR spectra of chlorpheniramine maleate

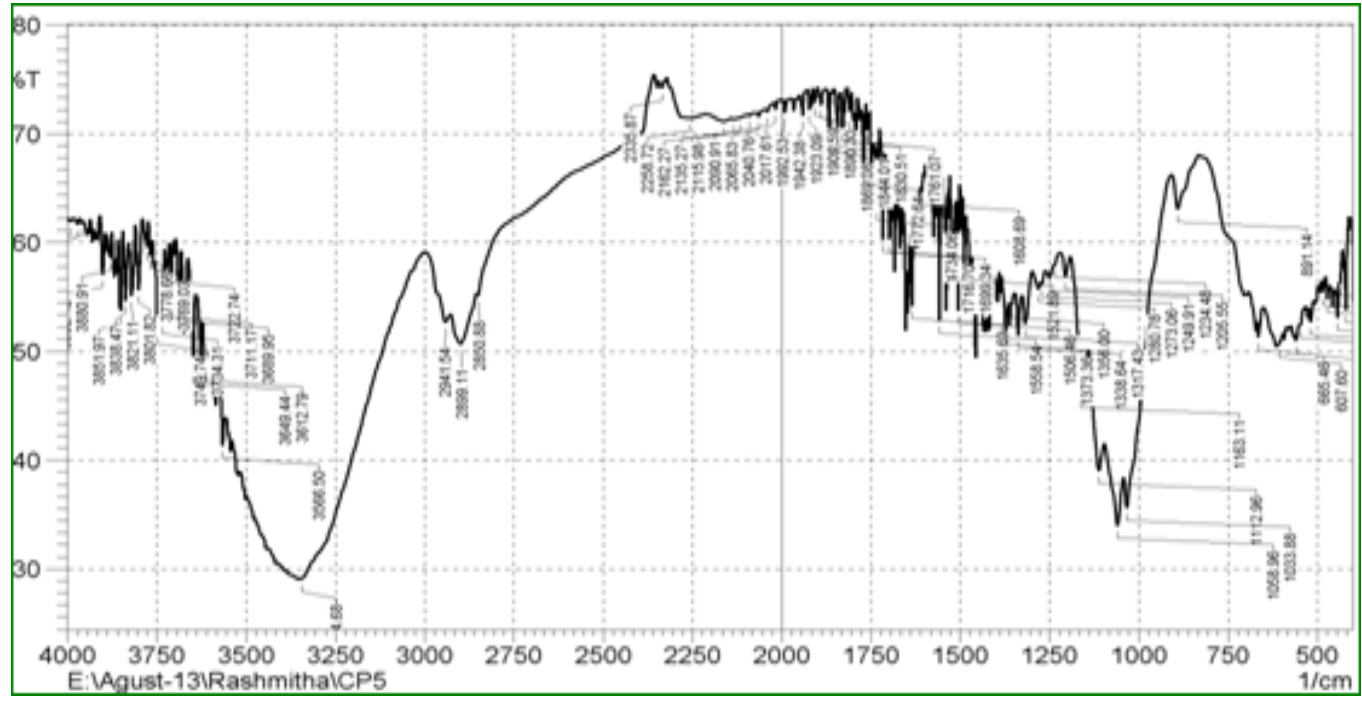

Fig. 5: FTIR spectra of medicated chocolate (chlorpheniramine maleate+chocolate base)

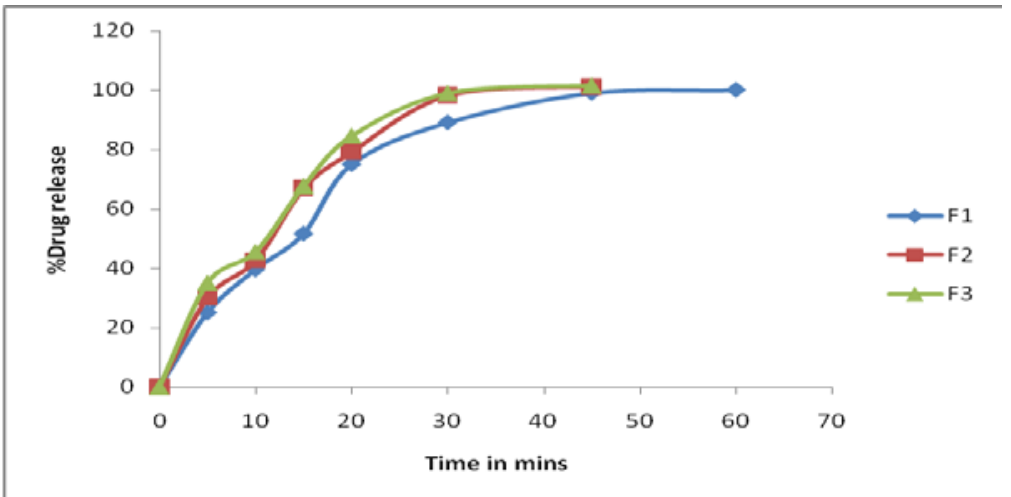

Fig. 6: Percentage cumulative drug release profile of chlorpheniramine maleate

\section{RESULTS}

\section{Construction of standard graph of chlorpheniramine maleate}

A standard graph of Chlorpheniramine Maleate was plotted with a slope of 0.090 and with a regression square of 0.997 as shown in the table 4 and fig. 1 . The formulation of the chocolate base is shown in the table 5 .

\section{Evaluation tests}

Evaluation of chocolate base

Taste, texture and mouth feel characteristics assessment

Taste, Texture and Mouth Feel Characteristics of chocolate base was determined by using 10 Human Volunteers and it was found to be 
satisfactory. It was scaled 1-5 under following characters which are shown in the table 6.

\section{Evaluation of medicated chocolate [10-13]}

\section{Differential scanning calorimetry}

The DSC spectra of Chlorpheniramine Maleate showed sharp endothermic peak at $103{ }^{\circ} \mathrm{C}$. DSC spectra of Medicated chocolate showed blunt endothermic peak at $104^{\circ} \mathrm{C}$. Which shows that, the drug is in stable form in the chocolate formulation and there are no drugexcipient interactions. The DSC spectra are shown in the fig. 2 and 3.

\section{Fourier transform infra red spectroscopy}

FTIR spectra of pure drug Chlorpheniramine Maleate showed bands at $1580 \mathrm{~cm}^{-1}$ due to $\mathrm{C}=\mathrm{C}$ stretching, $1476 \mathrm{~cm}^{-1}$ due to $\mathrm{C}-\mathrm{H}$ stretching and $1352 \mathrm{~cm}^{-1}$ due to $\mathrm{C}-\mathrm{H}$ bending, $864 \mathrm{~cm}^{-1}$ due to $\mathrm{C}-\mathrm{C}$ and $702 \mathrm{~cm}^{-1}$ due to and $\mathrm{C}-\mathrm{Cl}$ stretching vibration. The FTIR spectral bands are shown in the fig. 4 and 5 .

\section{General appearance}

Prepared formulations appear glossy and shiny. Prepared formulations are semi curvature in shape with length, width and height of 28.5, 18 and $14 \mathrm{~mm}$ respectively as shown in the table 7 .

\section{Thickness}

The thickness of the formulations was measured by using Vernier calipers. The mean thickness was almost uniform in all the batches and the values were found in the range of $8.99 \pm 0.02 \mathrm{~mm}-9.18 \pm 0.05$ $\mathrm{mm}$ as shown in the table 8 .

\section{Weight variation}

The Weight Variation study was performed according to USP as shown in the table 9. The average percentage deviations of all formulations were in the range of $3.06 \pm 0.24$ gms- $3.10 \pm 0.28$ gms respectively. The weights of all the formulations were almost uniform with low standard deviation values indicating effective mixing of the drug and excipients. The formulations showed no sign of sticking during preparation. Hence the formulations have passed the Weight Variation test.

\section{Disintegration test}

Disintegration Test for the prepared formulation was carried out as per USP shown in the table 10. The average time taken for the disintegration of formulations was almost uniform and they were in the range of $20 \pm 2 \mathrm{~min}-23 \pm 4$ min respectively.

\section{Drug content}

For Chlorpheniramine Maleate medicated chocolate square drug content was found to be $98.49 \pm 0.54 \mathrm{mg}$. The results are shown in the table 11.

\section{In vitro drug release (dissolution) [14]}

The chocolate formulation was subjected to in vitro drug release using 6.8 PH Buffer. Dissolution data shows that the first formulation releases complete drug within $60 \mathrm{~min}$. In time period of $5 \mathrm{~min}$, the drug release was found to be $25.10 \%$, in $10 \mathrm{~min}$ it was found to be $39.62 \%$, in $15 \mathrm{~min}$ it was found to be $51.66 \%$, in $20 \mathrm{~min}$ it was $75.06 \%$, in $30 \mathrm{~min}$ it was $89.05 \%$, in $45 \mathrm{~min}$ it was $99.00 \%$ and at last in $60 \mathrm{~min}$ it was found to be $100.00 \%$.

Dissolution data shows that the second formulation releases complete drug within $45 \mathrm{~min}$. In time period of $5 \mathrm{~min}$, the drug release was found to be $30.09 \%$, in $10 \mathrm{~min}$ it was found to be $42.62 \%$, in $15 \mathrm{~min}$ it was found to be $66.88 \%$, in $20 \mathrm{~min}$ it was $79.09 \%$, in $30 \mathrm{~min}$ it was $98.05 \%$, and at last in $45 \mathrm{~min}$ it was $100.99 \%$ and there is no drug release remained for release in 60 min. Dissolution data of the third formulation shows that it releases complete drug within $45 \mathrm{~min}$. In time period of $5 \mathrm{~min}$, the drug release was found to be $35.15 \%$, in $10 \mathrm{~min}$ it was found to be $45.62 \%$, in $15 \mathrm{~min}$ it was found to be $67.85 \%$, in $20 \mathrm{~min}$ it was $84.66 \%$, in $30 \mathrm{~min}$ it was $99.05 \%$, and at last in $45 \mathrm{~min}$ it was $101.62 \%$ and there is no drug release remained for release in 60 min. The dissolution data of all the formulations are shown in the table 12. The percentage cumulative drug release profile of Chlorpheniramine Maleate in all formulations is shown in the fig. 6.

\section{CONCLUSION}

It is concluded that, the Chlorpheniramine Maleate medicated chocolate formulations showed good drug release with $100 \%$ at 60 min of F1, with $100.99 \%$ at $45 \mathrm{~min}$ of F2 and $101.62 \%$ at 45 min of F3 respectively were observed. Drug excipient compatibility studies reveals that, the drug is stable and showed no drug-excipient interactions. Since, artificial sweetening agents are used, it can also be used by diabetic patients.

\section{CONFLICT OF INTERESTS}

Declare none

\section{REFERENCES}

1. Firriolo JF. Oral cavity: a review. Oral Surgery Med Oral Pathol 1994;78:189-93.

2. Sastry SV, Nyshdham JR. Review of formulation used in oral cavity. Pharm Sci Technol Today 2000;3:138-45.

3. Batheja P. Basic biopharmaceutics of buccal and sublingual absorption, enhancement in drug delivery. London, New York. Touitou E, Barry BW. editors. CRC Press: Taylor and Francis Group; 2006. p. 189.

4. Nidhi Patel, Saleha Diwan, Kajal Shukla, Priyanka Tomar, Hitesh Jain, Prasanna Pradhan, et al. Chocolate drug delivery system: a review. Indo Am J Pharm Sci 2015;2:1077-81.

5. Sharma M, Jain DK. Chocolate formulation as drug delivery system for pediatrics. Indonesian J Pharm 2012;23:216-24.

6. Lang KW. Delivery of active agents using a chocolate vehicle. US Patent 0269558; 2007.

7. Afoakwa EO, Paterson A, Fowler M. Chocolate science and technology. Wiley-Blackwell Publication 2008;226:1259.

8. Davide G, Cristina L, Stefano N, Giovambattista D, Claudio F. Short-term administration of dark chocolate is followed by a significant increase in insulin sensitivity and a decrease in blood pressure in healthy persons. Am J Clin Nutr 2005;81:611-4.

9. James F, Gerd P, Albert K. Effect of chocolate on acne vulgaris. J Am Med Assoc 1969;210:2071-4.

10. Sastry SV, Nyshadham JR, Fix JA. Recent technological advances in oral drug delivery-a review. Pharm Sci Technol Today 2000;3:138-44.

11. Knight Ian. Chocolate and Cocoa: Health and Nutrition. Blackwell Publication; 1999.

12. Rodgers C. The role of aversive bittering agents in the prevention of pediatric poisonings. Pediatrics 1994;93:68-9.

13. Chirag Vasani, Ketan Shah. Preparation and evaluation of chocolate drug delivery system of albendazole. Res J Pharm Tech 2016;9:1994-8.

14. Viswanath V, Narasimharao B, Purushothaman M, Sireesha S. Formulation and evaluation of fexofenadine hydrochloride and paracetamol chocolate for pediatrics. World J Pharm Res 2015;4:842-58.

\section{How to cite this article}

- $\quad$ Reddy Sunil, K Mounika, A Venkatesham. Design and fabrication of medicated chocolate formulation by chocolate drug delivery system. Int J Curr Pharm Res 2017;9(5):128-133. 